\title{
In-situ Correlative Analysis of Electrical and Mechanical Properties of 3D Nanostructures by Combination of AFM, SEM and FIB
}

Christian Schwalb ${ }^{1}$, Pinar Frank ${ }^{1}$, Stefan Hummel ${ }^{1}$, Juergen Sattelkov ${ }^{2}$, Robert Winkler ${ }^{2}$, Johanna Huetner ${ }^{1}$, Oleg Domanov ${ }^{1}$, Georg Fantner ${ }^{3}$ and Harald Plank ${ }^{2}$

${ }^{1}$ GETec Microscopy GmbH, Vienna, Wien, Austria, ${ }^{2}$ TU Graz, Graz, Steiermark, Austria, ${ }^{3}$ EPFL, Lausanne, Vaud, Switzerland

The combination of different analytical methods into one instrument is of great importance for the simultaneous acquisition of complementary information. Especially the in-situ combination of scanning electron microscopy (SEM) and atomic force microscopy (AFM) enables completely new insights in the micro and nano world. In this work, we present a unique AFM - the AFSEM - that can be easily integrated into the high-vacuum environment of almost any scanning electron microscope (SEM) or other host system (e.g. dual beam microscopes). It allows direct in-situ combination of these complementary techniques due to the simultaneous operation of SEM and AFM inside the vacuum chamber. Furthermore, due to the open design it can be combined with additional add-ons, e.g., tensile stages, nano-indentors or nano-manipulators. [1,2]

Especially the characterization of electrical properties of nanostructures inside the highvacuum environment of a SEM or FIB system is of great interest. We will present first results using the combination of novel self-sensing cantilevers [2] with functionalized tips for conductive, magnetic or thermal measurements, that are fabricated by the 3D nanoprinting technique using focused electron-beam induced deposition (FEBID).[4] We will show results for in-situ electrical characterization of nanostructures by combining SEM and conductive AFM measurements for dose-dependent conductivity measurements of nanogranular $\mathrm{Pt}(\mathrm{C})$ structures after focused electron beam induced processing (Figure 1b). In addition, we will present the in-situ electrical characterization of 2D materials as well as in-situ electric force microscopy (EFM) measurements on grain boundaries of $\mathrm{BaTiO}_{3}$.

In a further step, we demonstrate how in-situ correlative analysis with the AFSEM in an SEM can be extended into the third dimension to measure nanomechanical properties of soft materials. To achieve this, FIB slicing and mapping of nanomechanical properties using the AFSEM is performed in repetitive steps to build up a 3-dimensional elasticity map of, e.g., polymer blends (Figure 2).

Based on the broad variety of applications regarding the characterization of different materials and devices we anticipate the AFSEM to be one of the driving characterization tools for correlative SEM/FIB/AFM analysis in the future. 

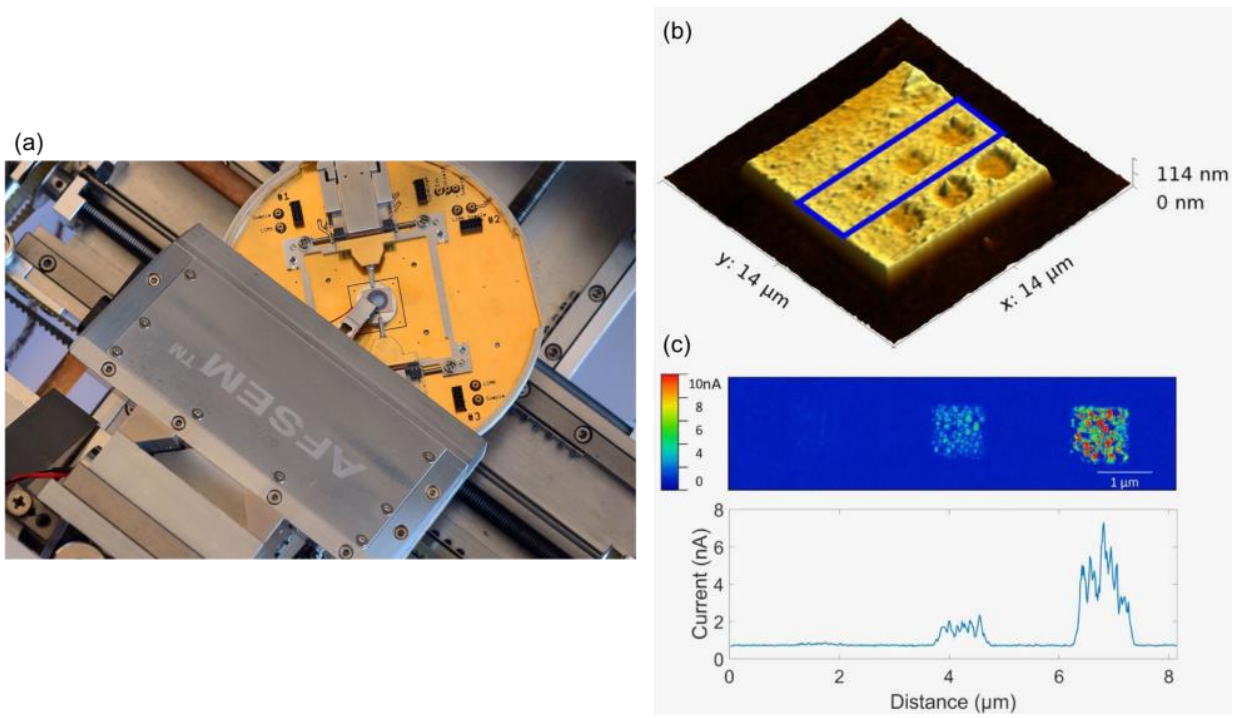

Figure 1. (a) AFSEM integration inside SEM in combination with a nanomanipulator setup. (b) In-situ AFM topography of nanogranular $\mathrm{Pt}(\mathrm{C})$ structure prepared by focused electron beam induced depostion. (c) In-situ conductive AFM measurements of nanogranular $\mathrm{Pt}(\mathrm{C})$ structure.
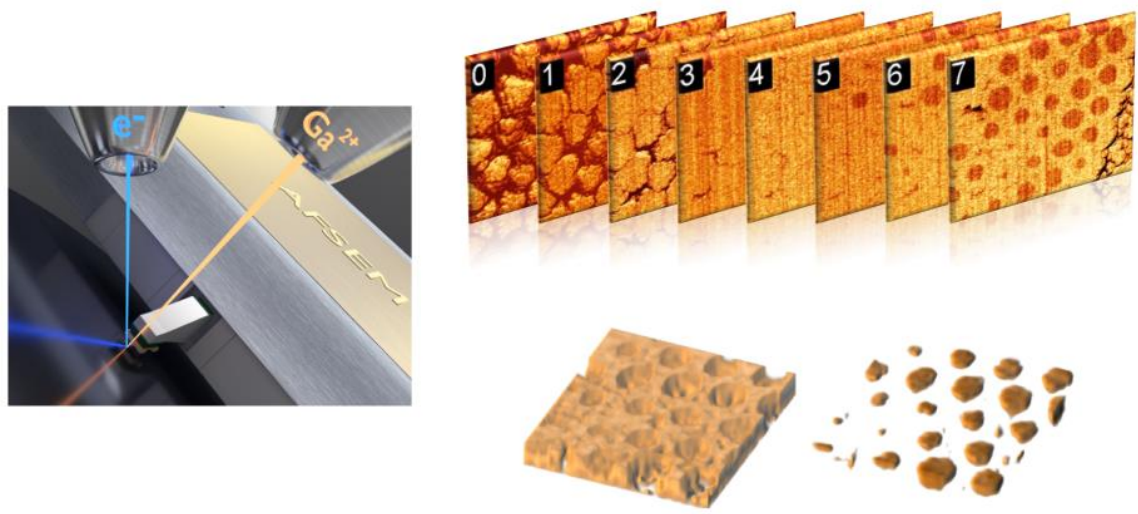

Figure 2. (Left) Schematics of correlative analysis in a dual-beam system using SEM, FIB and AFSEM in an interactive experiment. (Right top) Consecutive in-situ AFM elasticity maps of a polymer bead structure after different FIB slicing. (Right bottom) 3D reconstruction of nano-mechanical properties of the ion-beam treated polymer bead structure.References

[1] D. Yablon, P. Werten, M. Winhold and C.H. Schwalb, Microscopy and Analysis 31(2) 14-18 (2017)

[2] J. Kreith, T. Strunz, E.J. Fantner, G.E. Fantner and M.J. Cordill, Rev. Sci. Instr. 88 053704 (2017)

[3] M. Dukic, J. D. Adams and G.E. Fantner, Scientific Reports 5 (2015) 16393.

[4] H. Plank, R. Winkler, C. H. Schwalb, J. Hütner, J. D. Fowlkes, P. D. Rack, I. Utke, and M. Huth, Micromachines 11 (1), 48 (2020) 